\title{
Sleep Apnea and Cardiovascular Disease
}

\author{
Sadeka Tamanna ${ }^{1}$, M. Iftekhar Ullah ${ }^{2}$ \\ ${ }^{1}$ VA Sleep Lab, G.V. (Sonny) Montgomery VA Medical Center, Jackson, Mississippi, USA, ${ }^{2}$ Sleep \\ Medicine Fellowship Program, University of Mississippi Medical Center, Jackson, Mississippi, USA
}

(Cardiovasc.j. 2016; 8(2): 143-148)

\section{Keywords: \\ Sleep apnoea, Cardiovascular disease, Hypertension, heart failure.}

\section{Introduction:}

One third of human life is spent in sleep. Physiology of different systems in the body works under a different mode of central nervous system operation in sleep than in awake. Normal physiologic sleep consists of rapid eye movement (REM) sleep and nonrapid eye movement (NREM) sleep, which alternates within sleep cycles of about 90 minutes in length. Hemodynamic mechanisms change throughout these cycles of sleep due to various metabolic, endocrine, vascular and autonomic changes.

Sleep apnea is characterized by cessation of airflow during sleep. Depending on the mechanism of sleep apnea, it can be divided into two types: obstructive sleep apnea (OSA) and central sleep apnea (CSA). OSA is characterized by frequent upper airway collapse during sleep despite respiratory effort. CSA results from loss of respiratory effort leading to partial or complete cessation of breathing. OSA affects $17 \%$ to $24 \%$ of North American adults. ${ }^{1}$ The prevalence of OSA among patients with cardiovascular diseases is 3 to 4 fold higher than healthy persons. ${ }^{2}$ Cardiovascular diseases including hypertension, atrial fibrillation, congestive heart failure, stroke and sudden cardiac deaths are associated with OSA. In this review, we will discuss the interaction of sleep apnea with cardiovascular diseases and how to prevent cardiovascular complications among patients with comorbid OSA.

\section{Definition and diagnosis of OSA}

OSA is characterized by repetitive interruption of breathing caused by pharyngeal collapse during sleep. An obstructive apnea is defined as pause of breathing for more than 10 seconds with continued respiratory effort. Obstructive hypopneas are defined as reduction of airflow with fall in oxygen saturation. More than 30\% reduction of airflow for 10 seconds or more with $>3 \%$ desaturation from pre-event baseline is considered hypopnea. OSA is diagnosed when the patient has an apnea-hypopnea index (AHI; number of apneas and hypopneas per hour of sleep) $>5$ with the symptoms of excessive sleepiness during the day. ${ }^{3,4}$ The area of pharyngeal collapse in OSA is usually posterior to the tongue, uvula or soft palate or some combination of these structures. The diameter of the pharyngeal airway can be small from fat deposition in obesity or due to constriction of airway from arrangement of bone and surrounding soft tissue structures. In children, enlarged tonsils and adenoid are usually the cause of small airway. During wakefulness, the airflow resistance and turbulence can be handled by responsiveness of mechanoreceptors in the larynx causing increased activity of pharyngeal dilator muscles. During sleep, this reflex pharyngeal muscle activity is reduced or lost, leading to pharyngeal narrowing or intermittent collapse.

Diagnosis of OSA should start with screening of patients with high risk for OSA. Loud snoring, observed apneas, fragmented sleep, increased daytime sleepiness, unexplained fatigue and tiredness can be symptoms of OSA. A focused history with physical examination including neck size, oral examination with Mallampati score, BMI, hear rate, rhythm and blood pressure are needed during initial evaluation of sleep apnea. EPWORTH sleepiness scale, STOP BANG questionnaire, the Berlin Questionnaire and overnight oximetry may

Address of Correspondence: Dr. Sadeka Tamanna, VA Sleep Lab, G.V. (Sonny) Montgomery VA Medical Center, Jackson, Mississippi 39216, USA. Email: sadeka.tamanna@va.gov 
also be used for initial screening. Definitive diagnosis of sleep apnea requires an overnight polysomnography (PSG) or a home sleep study which record multiple physiological variables continuously during sleep. Overnight in-lab PSG channels generally include EEG for sleep staging, EMG for muscle activity, EOG (electro-oculogram) for eye movements, respiration (flow, effort, and oxygen saturation), snoring and a continuous lead of ECG.

\section{Central Sleep Apnea (CSA)}

A central apnea is defined as e" $90 \%$ reduction in breathing amplitude for at least 10 seconds with no respiratory effort. CSA syndrome is present when a patient has e" 5 central apnea per hour of sleep with associated symptoms of disrupted sleep (frequent arousals) and/or hypersomnolence during the day. CSA is observed in various disease conditions including congestive heart failure (CHF) and some neurological conditions including stroke. Cheyne-Stokes respiration is a form CSA seen frequently in $\mathrm{CHF}$, characterized by a crescendo decrescendo pattern of breathing with a central apnea or hypopnea at the nadir of respiratory effort. Diagnosis of CSA requires an overnight PSG to determine the frequency and pattern of central apnea.

\section{Pathophysiology of OSA leading to cardiovascular risk}

Sleep is characterized by cardiovascular quiescence with decrease in basal metabolic rate, heart rate, blood pressure and sympathetic activity and increase in vagal tone. Heart rate decreases during NREM sleep with a tonic increase in parasympathetic activity which fluctuates during REM sleep. Blood pressure (BP)generally drops by 5-14\% during NREM sleep and fluctuates during REM sleep. ${ }^{5}$ Cardiac output also decreases progressively during sleep, with the greatest decrease occurring during the last sleep cycle, particularly during last REM sleep (a physiologic phenomenon called "dipping"). OSA causes hemodynamic and autonomic instability by intermittent hypoxia, hypercapnia and heightened adrenergic activation from repeated apneic events. This exaggerated sympathetic drive elicited by apneas during sleep continue during the wakefulness period as well. ${ }^{6,7}$ OSA has also been linked to blunting or "non-dipping" of nocturnal blood pressure. A linear correlation has been found between 24 hour BP and AHI independent of BMI in the large cohort of Wisconsin Sleep Cohort Study. ${ }^{8}$ Heart rate variability is low and BP variability is high among patients with OSA. Combination of these two factors has been attributed to increase in cardiovascular risk and end organ damages from sleep apnea in several studies. ${ }^{5,9}$ (Figure 1)

\section{Mechanical effects of OSA:}

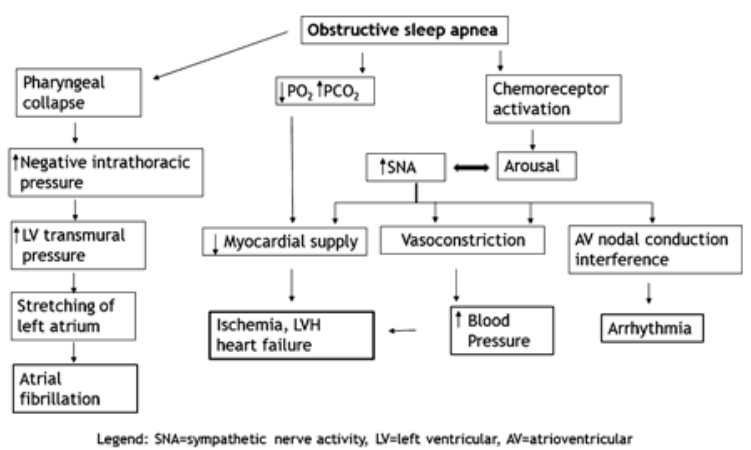

Fig.-1: Algorithm for pathophysiological effects of OSA. Adapted from Bradley et al., Circulation, $2003 .{ }^{34}$

During obstructive apneas, negative inspiratory intrathoracic pressure generated against the occluded pharynx increases left ventricular (LV) transmural pressure and thereby increases afterload. ${ }^{10,11}$ It also increases venous return, augmenting right ventricular preload, whereas OSA induced hypoxic pulmonary vasoconstriction increases pulmonary afterload. Consequently, RV distension and leftward septal displacement during diastole greatly impairs LV filling. This combination of increased LV afterload and diminished preload reduces stroke volume and cardiac output, which is more pronounced in heart failure patients than in healthy subjects. ${ }^{11}$ Increased LV transmural pressure also increases myocardial oxygen demand, while simultaneously reduction in coronary blood flow and hypoxia during an apneic event reduces oxygen delivery. This may lead to chronic myocardial ischemia, cardiac remodeling and heart failure in the long run. OSA was found to be associated with significant atrial remodeling characterized by atrial enlargement, reduction in voltage, site-specific and widespread conduction abnormalities, and longer sinus node recovery. ${ }^{12}$ Several studies showed that 
OSA predicts new onset atrial fibrillation (AF) or its recurrences following cardioversion to sinus rhythm. ${ }^{13,14}$

\section{Vascular inflammatory effects of OSA:}

Intermittent hypoxia in OSA induces oxygen free radicals and activates inflammatory pathways leading to vascular endothelial dysfunction. It also promotes oxidation of lipoproteins, increases expression of adhesion molecules and vascular smooth muscle proliferation. All these effects increase prevalence of hypertension and atherosclerosis in OSA. Platelet activation and adhesions are increased in patients with OSA during sleep, and these have been shown to decrease after one night of CPAP treatment. ${ }^{15} \mathrm{~A}$ randomized trial also demonstrated that treatment of OSA by CPAP reduces carotid intima-media thickness, supporting a cause effect relationship between atherosclerosis and OSA. ${ }^{16}$

\section{Association between OSA and cardiovascular diseases}

\section{OSA and hypertension:}

OSA has been found to be a significant predictor of cardiovascular diseases among middle aged population followed over seven years period $(\mathrm{OR}=4.9, \mathrm{CI}=1.8-13.6) .{ }^{17}$ The prevalence of OSA is higher in population with hypertension (30-83\%), ${ }^{18}$ heart failure (12-53\%), ${ }^{19}$ ischemic heart diseases $(30-58 \%)^{20}$ and stroke (43-91\%). ${ }^{21}$

Hypertension (HTN) has been extensively studied for its association with OSA. Prevalence of nondipping (drop of nocturnal mean arterial BP $>10 \%$ ) is very high among patients with moderate to severe sleep apnea, while not being treated with anti-hypertensive. ${ }^{22} \mathrm{~A}$ dose response relationships were found between AHI and hypertension inn Sleep Heart Health Study among 6132 participants. Excluding participants taking anti-hypertensive medications, AHI was linearly associated with blood pressure values in this study. ${ }^{23}$ Wisconsin Sleep Cohort Study prospectively studied 709 participants over 8 years and found 2.89 times $(95 \%$ $\mathrm{CI}=1.46-5.65)$ increased risk of developing HTN among participants with AHI of 5 to 15, after adjusting for age, sex, obesity and other comorbid factors. ${ }^{1}$ Resistant hypertension increases cardiovascular diseases by 1.5 fold, and patients with OSA have almost five fold higher risk of developing resistant HTN.${ }^{24}$ Multiple multi-centric randomized studies have shown improvement of resistant HTN with CPAP use. ${ }^{25-27}$ For obvious reasons, OSA has been included as an identifiable cause of hypertension since 2003 in JNC (Joint National Committee) report for blood pressure management. ${ }^{28}$

\section{OSA and heart failure:}

OSA is prevalent (49\%-53\%) among patients with CHF (LV ejection fraction <45\%). ${ }^{26,29}$ In a prospective study of 164 patients with $\mathrm{CHF}$, patients with untreated sleep apnea had significantly higher (hazard ratio $=2.89, p=0.029$ ) mortality than the patients with mild or no sleep apnea. ${ }^{30}$ Overall, untreated severe OSA significantly increase the risk of fatal $(\mathrm{OR}=2.87$, $95 \% \mathrm{CI}=1.17-7.75)$ and non-fatal(OR=3.17, 95\% $\mathrm{CI}=1.12-7.51$ ) cardiovascular events after adjusting for confounding factors. ${ }^{31}$ The causal role of OSA in development of CHF has been suggested by many clinical trials that showed improvement of cardiac function after treatment with CPAP. ${ }^{32-}$ ${ }^{34}$ Central sleep apnea, resulting in turn from $\mathrm{CHF}$, plays a major role in progressive morbidity and mortality among these patients. CSA is present in 25 to $40 \%$ patients with $\mathrm{CHF},{ }^{34}$ which results from cyclic hyperventilation and fall in partial pressure of carbon dioxide below the apnea threshold. This leads to tissue hypoxia, repetitive arousals from sleep and activation of the sympathetic nervous system, and independently increases the risk of mortality. CSA worsens the left atrial (LA) function more than OSA by decreasing the LA wall compliance. ${ }^{35}$ The CANPAP trial tested the effect of CPAP by randomizing patients of heart failure to CPAP and no-CPAP, and found that CPAP improves central apnea, nocturnal oxygenation, left ventricular ejection fraction and serum norepinephrine level. ${ }^{36}$ Adaptive servo ventilation (ASV) was introduced as a novel method of ventilator support designed for Cheyne Stokes Respiration for CHF. Multi centered randomized trials showed promising results with ASV improving left ventricular functions and sleep quality, compared to other modes of positive pressure ventilation. ${ }^{37}$ It is to be noted that a recent preliminary result (2015) of a multi-centered randomized trial (SERV-HF) on 1300 patients showed increased mortality among patients with 
CHF $(\mathrm{EF}<45 \%)$ who were treated with ASV for their CSA. ${ }^{38}$ Though final in-depth analysis of the study finding is yet to come, excess death appeared to be driven by sudden cardiac deaths.

\section{OSA and Atrial Fibrillation:}

Approximately $20-50 \%$ of patients with atrial fibrillation (AF) have OSA.OSA is more prevalent in patients with $\mathrm{AF}$ than in high-risk patients with multiple other cardiovascular diseases (49\% versus $32 \%, \mathrm{P}=0.0004$ ) and the odds of having $\mathrm{AF}$ in patients with OSA is about 2.19 (95\% CI 1.40 -3.42, $\mathrm{P}=0.0006) .{ }^{39}$ Patients with OSA are likely to be more symptomatic $(p=0.001)$ and be on rhythm control therapy than patients without OSA $(\mathrm{p}=0.0037) .{ }^{40}$ The diagnosis (hazard ratio $=1.55 ; 95 \%$ $\mathrm{CI}=1.21-2.00)$ and severity $(\mathrm{HR}=1.15 ; 95 \%$ $\mathrm{CI},=1.06-1.26)$ of OSA have been reported to be independent predictors of hospitalization for incident atrial fibrillation. ${ }^{41}$ Risk of recurrence of $\mathrm{AF}$ is two-fold among patients with untreated OSA compared to those who has been compliant with their CPAP therapy $(\mathrm{p}=0.013) .{ }^{14}$ CPAP treated patients are also twice as likely to have AF-free survival after catheter ablation compared to those not treated with CPAP (71.9\% vs. $36.7 \%$; $=0.01) .{ }^{42}$

\section{OSA and Stroke:}

The prevalence of OSA is high (up to 60\%) among patients with stroke. ${ }^{43-46}$ Incompletely treated OSA increases the risk of CVD by 11 folds compared to those treated adequately with CPAP. AHI and hypoxemia are strongly correlated with the prevalence of CVD with relative odds of 2.1 to 2.5 for moderate to severe sleep apnea. ${ }^{47}$ In Sleep Heart Health Study, a total of 5,422 participants without a history of stroke at baseline and untreated for sleep apnea were followed for a median of 8.7 years. One hundred ninety-three ischemic strokes were observed in this population, and a significant positive association was found with ischemic stroke with a linear increase of AHI $(\mathrm{P}=0.016){ }^{48}$ Yaggi et al. followed a cohort of 1022 patients who underwent PSG and were followed for subsequent events of stroke or death. After adjusting for age, sex, dyslipidemia, HTN, DM, atrial fibrillation, OSA retained a statistically significant association with development of stroke and death $(\mathrm{HR}=1.97, \mathrm{P}=0.01) .{ }^{43}$

\section{Sudden death from cardiovascular diseases:}

Clinical evidence of sudden cardiac death induced by OSA has been addressed in different studies. Yumino et al., in a prospective cohort study of 89 patients with ACS who underwent polysomnography, found OSA in $57 \%$ of patients. After a mean follow up of $>7$ months, the incidence of cardiac death, re-infarction or new revascularization was higher in patients with OSA compared to those without OSA. ${ }^{49}$ People with OSA suffer from sudden death more frequently while sleeping than awake. An observational study on 112 residents of Minnesota who died suddenly from a cardiovascular event showed that $64 \%$ of patients with OSA died between midnight to 6 AM compared to $21 \%$ people without OSA $(\mathrm{P}=0.01), 16 \%$ of general population $(\mathrm{P}<0.001)$, and $25 \%$ expected by chance. For people with OSA, relative risk of sudden death from cardiovascular event occurring between midnight to $6 \mathrm{AM}$ was 2.57 (95\% CI =1.873.52)..$^{50}$

Mechanism of sudden cardiac death in OSA was explored in a recent study. Microvolt $\mathrm{T}$ wave alternant (MTWA) was used as a tool to measure myocardial vulnerability to assess the potential arrhythmogenicity of sleep apnea on causing sudden deaths. The severity of sleep apnea (AHI) was directly proportional to the presence of MTWA, suggestive of the direct effect of OSA on sudden death. ${ }^{51}$ OSA may lead to to higher incidence of sudden cardiac death due to reentrant arrhythmia initiated by ventricular extra-systole that excite myocardial fibers at the height of its vulnerable period, and reentrant beats trigger runs of ventricular tachycardia and ventricular fibrillation.

\section{Conclusion:}

Obstructive sleep apnea may increase the risk of cardiovascular morbidity and mortality by various mechanisms. Repetitive nocturnal hypoxemia and arousals lead to increased risk of atherosclerosis and hypercoagulability, cardiac ischemia, cellular degeneration, and apoptosis. It may also promote cardiac remodeling causing diastolic and systolic dysfunctions; which provide fertile environment for reentrant arrhythmias. Since the prevalence of obstructive sleep apnea is fairly high, screening for this condition in vulnerable population with early detection and treatment may help prevent 
cardiovascular complication in this group of patients.

\section{Conflict of Interest - None.}

\section{References:}

1. Young T, Peppard PE, Gottlieb DJ. Epidemiology of obstructive sleep apnea: a population health perspective. Am J Resp Crit Care 2002;165(9):1217-1239.

2. Wolk R, Kara T, Somers VK. Sleep-disordered breathing and cardiovascular disease. Circulation 2003;108(1):9-12.

3. Schechter MS. Technical report: diagnosis and management of childhood obstructive sleep apnea syndrome. Pediatrics 2002;109(4):e69.

4. Task A academy of sleep medicine. Sleep-related breathing disorders in adults: recommendations for syndrome definition and measurement techniques in clinical research. The Report of an American Academy of Sleep Medicine Task Force. Sleep 1999;22(5):667-689.

5. Baumgart P. Circadian Rhythm of Blood Pressure: Internal and External Time Triggers. Chronobiol Int 1991; 8(6):1, 444-450.

6. Somers VK, Mark AL, Zavala DC, Abboud FM. Contrasting effects of hypoxia and hypercapnia on ventilation and sympathetic activity in humans. J Appl Physiol 1989;67(5):2101-2106.

7. Somers VK, Dyken ME, Clary MP, Abboud FM. Sympathetic neural mechanisms in obstructive sleep apnea. J Clin Invest 1995;96(4):1897-1904.

8. Young T. Population-Based Study of Sleep-Disordered Breathing as a Risk Factor for Hypertension. Arch Intern Med 1997;157(15):1746.

9. Narkiewicz K, Montano N, Cogliati C, van de Borne PJH, Dyken ME, Somers VK. Altered Cardiovascular Variability in Obstructive Sleep Apnea. Circulation 1998;98(11):1071-1077.

10. Bradley T, Hall M. Hemodynamic effects of simulated obstructive apneas in humans with and without heart failure. Chest 2001.

11. Parker JD, Brooks D, Kozar LF, et al. Acute and chronic effects of airway obstruction on canine left ventricular performance. Am J Resp Crit Care 1999;160(6):1888-1896.

12. Dimitri H, Ng M, Brooks AG, et al. Atrial remodeling in obstructive sleep apnea: implications for atrial fibrillation. Heart Rhythm / 2012;9(3):321-327.

13. Gami AS, Hodge DO, Herges RM, et al. Obstructive sleep apnea, obesity, and the risk of incident atrial fibrillation. J Am Coll Cardiol 2015 2007;49(5):565-571.

14. Kanagala R, Murali NS, Friedman PA, et al. Obstructive sleep apnea and the recurrence of atrial fibrillation. Circulation 2003;107(20):2589-2594.

15. Bokinsky G. Spontaneous Platelet Activation and Aggregation During Obstructive Sleep Apnea and Its
Response to Therapy With Nasal Continuous Positive Airway Pressure. Chest 1995;108(3):625.

16. Drager LF, Bortolotto LA, Figueiredo AC, Krieger EM, Lorenzi GF. Effects of continuous positive airway pressure on early signs of atherosclerosis in obstructive sleep apnea. Am J Resp Crit Care 2007;176(7):706-712.

17. Peker Y, Hedner J, Norum J, Kraiczi H, Carlson J. Increased incidence of cardiovascular disease in middleaged men with obstructive sleep apnea: a 7-year followup. Am J Resp Crit Care 2002;166(2):159-165.

18. Logan AG, Perlikowski SM, Mente A, et al. High prevalence of unrecognized sleep apnoea in drugresistant hypertension. J Hypertens 2001;19(12):22712277.

19. Chan J, Sanderson J, Chan W, et al. Prevalence of sleep-disordered breathing in diastolic heart failure. Chest 1997;111(6):1488-1493.

20. Mooe T, Franklin KA, Holmström K, Rabben T, Wiklund U. Sleep-disordered breathing and coronary artery disease: long-term prognosis. Am J Resp Crit Care 2001;164(10 Pt 1):1910-1913.

21. Bassetti C, Aldrich MS. Sleep apnea in acute cerebrovascular diseases: final report on 128 patients. Sleep 1999;22(2):217-223.

22. Loredo J. Sleep quality and blood pressure dipping in obstructive sleep apnea. Am J Hypertens 2001;14(9):887892.

23. Young T, Shahar E, Nieto FJ, et al. Predictors of sleepdisordered breathing in community-dwelling adults: the Sleep Heart Health Study. Arch Intern Med 2002;162(8):893-900.

24. Gonçalves SC, Martinez D, Gus M, et al. Obstructive sleep apnea and resistant hypertension: a case-control study. Chest 2007;132(6):1858-1862.

25. Pedrosa RP, Drager LF, de Paula LKG, Amaro ACS, Bortolotto LA, Lorenzi-Filho G. Effects of OSA treatment on BP in patients with resistant hypertension: a randomized trial. Chest 2013;144(5):1487-1494.

26. Javaheri S. Sleep disorders in systolic heart failure: a prospective study of 100 male patients. The final report. Int $J$ Cardiol 2006;106(1):21-28.

27. de Oliveira AC, Martinez D, Massierer D, et al. The antihypertensive effect of positive airway pressure on resistant hypertension of patients with obstructive sleep apnea: a randomized, double-blind, clinical trial. Am J Resp Crit Care 2014;190(3):345-347.

28. Chobanian A V, Bakris GL, Black HR, et al. The Seventh Report of the Joint National Committee on Prevention, Detection, Evaluation, and Treatment of High Blood Pressure: the JNC 7 report. JAMA 2003;289(19):25602572.

29. Ferrier K, Campbell A, Yee B, et al. Sleep-disordered breathing occurs frequently in stable outpatients with congestive heart failure. Chest 2005;128(4):2116-2122. 
30. Wang H, Parker JD, Newton GE, et al. Influence of obstructive sleep apnea on mortality in patients with heart failure. J Am Coll Cardiol 2015 2007;49(15):16251631.

31. Marin JM, Carrizo SJ, Vicente E, Agusti AG. Longterm cardiovascular outcomes in men with obstructive sleep apnoea-hypopnoea with or without treatment with continuous positive airway pressure: an observational study. Lancet 2005;365(9464):1046-1053.

32. Arias MA, García-Río F, Alonso-Fernández A, Mediano O, Martínez I, Villamor J. Obstructive sleep apnea syndrome affects left ventricular diastolic function: effects of nasal continuous positive airway pressure in men. Circulation 2005;112(3):375-383.

33. Kaneko Y, Floras JS, Usui K, et al. Cardiovascular effects of continuous positive airway pressure in patients with heart failure and obstructive sleep apnea. $N$ Engl J Med 2003;348(13):1233-1241.

34. Bradley TD. Sleep Apnea and Heart Failure: Part II: Central Sleep Apnea. Circulation 2003;107(13):18221826.

35. Haruki N, Tsang W, Thavendiranathan P, et al. Impact of Sleep Apnea Phenotype on left Atrial Structure and Function in Systolic Heart Failure.. J Am Coll Cardiol 2015;65(10):A821.

36. Bradley TD, Logan AG, Kimoff RJ, et al. Continuous positive airway pressure for central sleep apnea and heart failure. N Engl J Med 2005;353(353):2025-2034.

37. Kasai T, Usui Y, Yoshioka T, et al. Effect of flowtriggered adaptive servo-ventilation compared with continuous positive airway pressure in patients with chronic heart failure with coexisting obstructive sleep apnea and Cheyne-Stokes respiration. Circ Heart Fail 2010;3(1):140-148.

38. Ayas NT, Patil SP, Stanchina M, Malhotra A. Treatment of Central Sleep Apnea with Adaptive Servoventilation in Chronic Heart Failure. Am J Resp Crit Care July 2015; 192:132-133.

39. Gami AS, Pressman G, Caples SM, et al. Association of atrial fibrillation and obstructive sleep apnea. Circulation 2004;110(4):364-367.

40. Holmqvist F, Guan N, Zhu Z, et al. Impact of obstructive sleep apnea and continuous positive airway pressure therapy on outcomes in patients with atrial fibrillationResults from the Outcomes Registry for Better
Informed Treatment of Atrial Fibrillation (ORBIT-AF). Am Heart J 2015;169(5):647-654.

41. Cadby G, McArdle N, Briffa T, et al. Severity of OSA Is an Independent Predictor of Incident Atrial Fibrillation Hospitalization in a Large Sleep-Clinic Cohort. Chest 2015;148(4):945-952.

42. Fein AS, Shvilkin A, Shah D, et al. Treatment of obstructive sleep apnea reduces the risk of atrial fibrillation recurrence after catheter ablation. J Am Coll Cardiol 2013;62(4):300-305.

43. Yaggi HK, Concato J, Kernan WN, Lichtman JH, Brass LM, Mohsenin V. Obstructive sleep apnea as a risk factor for stroke and death. $N$ Engl J Med. 2005;353(19):2034-2041.

44. Munoz R, Duran-Cantolla J, Martínez-Vila E, et al. Severe sleep apnea and risk of ischemic stroke in the elderly. Stroke 2006;37(9):2317-2321.

45. Bassetti CL, Milanova M, Gugger M. Sleep-disordered breathing and acute ischemic stroke: diagnosis, risk factors, treatment, evolution, and long-term clinical outcome. Stroke 2006;37(4):967-972.

46. Sahlin C, Sandberg O, Gustafson Y, et al. Obstructive sleep apnea is a risk factor for death in patients with stroke: a 10-year follow-up. Arch Intern Med 2008;168(3):297-301.

47. Shahar E, Whitney CW, Redline S, et al. Sleepdisordered breathing and cardiovascular disease: crosssectional results of the Sleep Heart Health Study. Am J Resp Crit Care 2001;163(1):19-25.

48. Redline S, Yenokyan G, Gottlieb DJ, et al. Obstructive sleep apnea-hypopnea and incident stroke: the sleep heart health study. Am $J$ Resp Crit Care 2010;182(2):269-277.

49. Yumino D, Tsurumi Y, Takagi A, Suzuki K, Kasanuki H. Impact of obstructive sleep apnea on clinical and angiographic outcomes following percutaneous coronary intervention in patients with acute coronary syndrome. Am J Cardiol 2007;99(1):26-30.

50. Gami AS, Howard DE, Olson EJ, Somers VK. Daynight pattern of sudden death in obstructive sleep apnea. N Engl J Med 2005;352(12):1206-1214.

51. Chan A, Antonio N. Mechanism of sudden cardiac death in obstructive sleep apnea, revisited. Sleep Medicine. 2013;14:e95. 